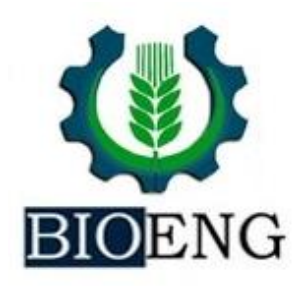

\title{
PROPRIEDADES FÍSICO-HÍDRICAS DE UM LATOSSOLO ARGILOSO APÓS COMPACTAÇÃO INDUZIDA MECANICAMENTE
}

${ }^{1}$ Centro Universitário Assis Gurgacz, Curso de Agronomia, Cascavel, PR, Brasil

${ }^{2}$ UNIOESTE- Universidade Estadual do Oeste do Paraná, Programa de Pós-graduação em Engenharia de Energia na Agricultura, Cascavel, PR, Brasil

Article history: Received 10 August 2017; Received in revised form 25 September 2017; Accepted 26 September 2017; Available online 29 September 2017.

\section{RESUMO}

A compactação do solo é um dos principais fatores que altera a qualidade do solo e o crescimento das culturas. Objetivou-se avaliar o efeito da compactação sobre as propriedades físicas - hídricas do solo. O delineamento experimental inteiramente casualizado (DIC) foi composto por 4 tratamentos e 5 repetições (T1 - sem compactação; T2 - quatro passadas com trator; T3 - seis passadas com trator e T4 - oito passadas com trator) a compactação foi realizada no dia da coleta de dados. As amostras foram submetidas às tensões $-0,033$ e $-0,05$ MPa em câmara de pressão de Richards. Com a utilização de amostras indeformadas, foi utilizado o equipamento psicrômetro WP4-T. Os dados foram submetidos à análise de variância (ANOVA) e as médias dos tratamentos foram comparadas pelo teste Tukey a 5\% de significância, pelo Software Assistat. Em relação à Porosidade total $(\mathrm{Pt})$, na profundidade de $10-20 \mathrm{~cm}$ o tratamento T3 apresentou menores valores. Conclui-se que a quantidade de passadas de um trator, em um Latossolo argiloso, alterou as características físico-hídricas do solo, com exceção da microporosidade. A camada de $10-20 \mathrm{~cm}$ foi a que apresentou maiores diferenças estatísticas, com alterações nos valores de Pt, Macroporos e DTA.

Palavras-chave: Armazenamento hídrico, porosidade, estrutura do solo.

\section{PHYSICAL - WATER A CLAYEY OXISOL AFTER MECHANICALLY INDUCED COMPRESSION}

\begin{abstract}
Soil compaction is one of the main factors that change the quality of soil and crop growth. The experiment will be conducted to evaluate the effect of compression on the physical - soil water. The completely randomized design (CRD) will be composed of 4 treatments and 5 repetitions (T1 - without compression; T2 - four passed with tractor, T3 - past six with tractor and T4 - eight strides with tractor) compression was held on the data collect. The samples submitted to tensions 33 and $100 \mathrm{kPa}$ Richards pressure chamber. To obtain the values of the total porosity of the soil, samples were prepared and packaged in plastic trays, and saturated in water. Data were submitted to analysis of variance (ANOVA) and treatment means were compared by the test "Tukey 5\%" of significance, the Assistat Software. In relation to Pt, at the depth of 10-20 cm T3 treatment had lower values. It is concluded that the amount of pasts of a tractor, in a clay latosol, altered the physical-water characteristics of the soil, except for
\end{abstract}

\footnotetext{
*silenetais@outlook.com
} 
the microporosity. The $10-20 \mathrm{~cm}$ layer presented the highest statistical differences, with changes in Pt, Macroporos and DTA values.

Keywords: water storage, porosity, soil structure.

\section{INTRODUÇÃO}

A compactação do solo pode ser entendida como a diminuição de volume do solo, o mesmo altera sua estrutura, normalmente solos que tem partículas menores são mais fáceis de serem afetados (CRISPIM, 2007).

A curva de retenção de água do solo (CRA) expressa a relação entre a umidade do solo, com base em massa ou volume. $\mathrm{O}$ conhecimento deste parâmetro é extremamente importante nos estudos que envolvam água no solo. Com base nesses pares de dados, é traçada uma curva para representar as características de retenção de água do solo, ou seja, a correlação entre a umidade e a tensão com quem a água esta retida nele. (GRIGOLON, 2013)

Segundo Passos (2004), o espaço poroso do solo exerce grande importância na movimentação da água, do ar e no crescimento das raízes, sofre grande alteração com o preparo do solo. Com o revolvimento do solo e o tráfego de máquinas e implementos agrícolas contribuem para alteração da estrutura do solo, modificando o tamanho dos agregados, aumentando a densidade do solo, reduzindo a porosidade total e aumentando à distribuição de microporos em relação aos macroporos, como consequência afeta a condutividade hidráulica saturada.

Nos últimos anos o indicativo de qualidade e sobre a penetração de água no solo tem sido extremamente de grande importância, o mesmo indica uma qualidade física de solo para a produção das culturas. Um dos objetivos é estabelecer uma relação quantitativa entre a compactação de solo e sobre a produção de culturas. (GUBIANI, 2012)

A CRA no solo é um atributo físico com várias interações e atuações de outros atributos do solo. Diante disso, pode ser considerado um indicador de qualidade física do solo e está relacionada diretamente com o desenvolvimento dos vegetais, devido à variação de umidade no solo influenciar na resistência mecânica do solo à penetração, na aeração, na condutividade hidráulica e no potencial matricial de água (DAVALO, 2013).

Segundo Nascimento (2009), uma das importâncias da CRA é o auxílio ao manejo da irrigação para os produtores sendo possível estimar a quantidade de água disponível para a planta, porém é de difícil caracterização, principalmente devido às amostras precisarem de uma boa condição estrutural e pelo longo tempo para obtenção dos resultados.

A CRA é utilizada para estudos tanto no balanço de aguas no solo, quanto na disponibilidade de agua nas plantas, e também sobre a infiltração da água. A partir da mesma podemos obter a capacidade de campo (CC), o ponto de murcha (PM) entre outros. (BARRETO, 2011). A determinação da capacidade de campo, do ponto de murcha permante e sobre a quantidade de agua disponível são inúmeras interferências de importância, desde que tenha o conhecimento de curva de retenção de agua no solo. (GRIGOLON, 2013).

Os valores de Capacidade de campo (CC) e Ponto de murcha permanente (PMP) podem ser encontrados com a utilização da câmara de Richards, ou com o psicrômetro WP4-T. Segundo Klein et al. (2010), a utilização da câmara de Richards, possui um complexo sistema pneumático, as placas porosas são frágeis e nem sempre é obtido o contato perfeito entre a amostra de solo e a placa. Outro problema é a demora nas determinações em função do lento equilíbrio do potencial da água na amostra. Já o psicrômetro WP4-T é um equipamento versátil e pode ser utilizado para determinar o PMP, mas apresenta 
resultados pouco precisos para potenciais maiores que -1,0 MPa.

Sendo assim, o presente trabalho objetivou avaliar o efeito de diferentes

\section{MATERIAL E MÉTODOS}

O presente trabalho foi realizado no município de Cascavel, localizado na região oeste do estado do Paraná, no Centro Universitário Assis Gurgacz (FAG), com as coordenadas geográficas "latitude $24^{\circ} 57^{\prime} 21^{\prime}$ " e longitude ' $53^{\circ} 27^{\prime}$ 19" e altitude de $781 \mathrm{~m}$, e solo classificado como Latossolo vermelho (EMBRAPA, 2013).

O clima é subtropical mesotérmico superúmido (KÖPPEN, 1936), com temperatura média anual em torno de $19{ }^{\circ} \mathrm{C}$. A temperatura máxima média em janeiro é de $28,6^{\circ} \mathrm{C}$, e em julho a mínima média é de $11,2^{\circ} \mathrm{C}$, com ocorrência de geadas. Pluviosidade média anual de 1248 $\mathrm{mm}$.

$\mathrm{O}$ delinamento experimental utilizado foi interamente casualizado (DIC). Foram coletadas amostras com estrutura preservada utilizando cilindros metálicos de $0,05 \mathrm{~m}$ de diâmetro e $0,04 \mathrm{~m}$ de altura, nos horizontes superficiais (0 $10 \mathrm{~cm} ; 10-20 \mathrm{~cm}$ e $20-30 \mathrm{~cm}$ ) dos perfis estudados. Foram realizadas cinco repetições em cada tratamento (T0 - sem compactação; T1 - quatro passadas com trator; T2 - seis passadas com trator e T3 oito passadas com trator).

A coleta de amostras ocorreu em área homogênea, onde foram criadas as faixas de compactação com um trator Massey Ferguson da Série MF 7180 de peso com lastro $9.900 \mathrm{~kg}$.

Para a obtenção dos valores de porosidade total $(\mathrm{Pt})$, as amostras foram preparadas e acondicionadas em bandejas plásticas, e saturadas em água durante um período de 24 horas. Posteriormente, as amostras foram levadas para uma coluna

\section{RESULTADOS E DISCUSSÃO}

Os dados referentes à $\mathrm{Pt}$ dos diferentes tratamentos de compactação níveis de compactação sobre propriedades físico - hídricos do solo.

de areia a -0,006 MPa de tensão de sucção por 48 horas, com objetivo de retirar a água contida nos macroporos. Após serem pesadas em balança de precisão de $0,1 \mathrm{~g}$ de exatidão, foram colocadas em estufa à 105 ${ }^{\circ} \mathrm{C}$ por 48 horas, para posterior quantificação da microporosidade (REINERT e REICHERT, 2006).

Para o cálculo da $\mathrm{Pt}$, foi realizada análise de densidade de partículas segundo metodologia do balão volumétrico com álcool (EMBRAPA, 1997).

Após as amostras ficarem $24 \mathrm{~h}$ em saturação, essas foram submetidas às tensões de -0,033 e -0,05 MPa em câmara de pressão de Richards, como descrito pela Embrapa (2011), utilizando amostras indeformadas, com posterior utilização de psicrômetro WP4-T, para as medidas de maiores pressões de sucção, utilizando-se amostras de $2 \mathrm{~g}$ de solo para configurar os demais pontos da curva de retenção de água do solo.

Para o cálculo da disponibilidade total de água no solo (DTA), utilizou-se a equação 1.

$$
D T A=\left(\frac{C c-P m}{10}\right) * D s \quad \text { (Equação 1) }
$$

Cc: Capacidade de campo (\%)

Pm: Ponto de murcha $(\%)$

Ds: Densidade do solo $\left(\mathrm{g} \mathrm{cm}^{-3}\right)$

Os dados de Pt, macroporos, microporos e DTA, foram submetidos à análise de variância (ANOVA) e as médias dos tratamentos comparadas pelo teste Tukey a 5\% de significância, pelo software ASSISTAT (SILVA e AZEVEDO, 2016).

induzida estão descritos na Tabela 1 . Verifica-se que para a primeira camada de 
0-10 cm não houve diferença estatística entre os tratamentos. Na camada de 10-20 $\mathrm{cm}$, o tratamento $\mathrm{T} 3$ apresentou menor valor de $\mathrm{Pt}$, sendo diferente do T4, e semelhante aos demais tratamentos.
Entretanto, T1, T2 e T4 foram considerados estatisticamente iguais. $\mathrm{Na}$ última camada analisada, não foram encontradas diferenças significativas entre os tratamentos.

Tabela 1 - Porosidade total (\%) em três profundidades referentes à testemunha e 3 níveis de compactação induzidas com trator.

\begin{tabular}{llll}
\hline Tratamentos & \multicolumn{3}{c}{ Profundidades } \\
\hline & $\mathbf{0} \mathbf{- 1 0} \mathbf{~ c m}$ & $\mathbf{1 0} \mathbf{- 2 0} \mathbf{~ c m}$ & $\mathbf{2 0}-\mathbf{3 0} \mathbf{~ c m}$ \\
T1 & $55,31 \mathrm{a}$ & $54,87 \mathrm{ab}$ & $58,37 \mathrm{a}$ \\
$\mathrm{T} 2$ & $56,67 \mathrm{a}$ & $56,16 \mathrm{ab}$ & $58,43 \mathrm{a}$ \\
$\mathrm{T} 3$ & $58,14 \mathrm{a}$ & $53,51 \mathrm{~b}$ & $58,28 \mathrm{a}$ \\
T4 & $57,21 \mathrm{a}$ & $59,31 \mathrm{a}$ & $56,84 \mathrm{a}$ \\
\hline DMS & 7,15 & 5,11 & 5,12 \\
CV \% & 6,94 & 5,04 & 4,88 \\
\hline
\end{tabular}

Médias de tratamentos seguidas por letras distintas na coluna diferem pelo Teste tukey a $5 \%$ de significância

$\mathrm{Cv}=$ Coeficiente de variação; DMS= Diferença Mínima Significativa.

(T1 - sem compactação; T2 - quatro passadas com trator; T3 - seis passadas com trator e T4 oito passadas com trator)

O presente trabalho diverge dos resultados de Mioranza et al. (2015), que trabalhando com diferentes níveis de compactação em um latossolo argiloso, constaram que a camada $0-10 \mathrm{~cm}$ diferiu dos tratamentos com duas e seis passadas com trator agrícola.

Bonini et al. (2011), encontraram diferenças estatísticas entre os tratamentos (1, 3, 5 passadas) com rolo compactador e a testemunha (sem passadas), para as 3 camadas avaliadas.

Dados referente a macroporosidade do solo estão descritos na tabela 2 . Verifica- se que para a primeira camada de
0-10 $\mathrm{cm}$ houve diferença estatística entre T3 que apresentou menor valor de macroporos, sendo diferente do $\mathrm{T} 4 \mathrm{e}$ semelhante aos demais tratamentos. No entanto, T1, T2 e T4 foram considerados estatisticamente iguais. Verifica- se que para a camada de $10-20 \mathrm{~cm}$ houve diferença estatística entre T2 que apresentou menor valor de macroporos, sendo diferente do T4, e semelhante aos demais tratamentos, no entanto, T1, T3 e $\mathrm{T} 4$ foram considerados estatisticamente iguais. Para a última camada de $20-30 \mathrm{~cm}$ não houve diferença estatística entre os tratamentos.

Tabela 2 - Macroporos (\%) do solo em três profundidades referentes à testemunha e 3 níveis de compactação induzidas com trator.

\begin{tabular}{llll}
\hline Tratamentos & \multicolumn{3}{c}{ Profundidades } \\
\hline & $\mathbf{0} \mathbf{- 1 0} \mathbf{~ c m}$ & $\mathbf{1 0} \mathbf{- 2 0} \mathbf{~ c m}$ & $\mathbf{2 0}-\mathbf{3 0} \mathbf{~ c m}$ \\
T1 & $20,99 \mathrm{ab}$ & $23,35 \mathrm{ab}$ & $22,26 \mathrm{a}$ \\
$\mathrm{T} 2$ & $21,28 \mathrm{ab}$ & $18,03 \mathrm{~b}$ & $22,19 \mathrm{a}$ \\
$\mathrm{T} 3$ & $17,21 \mathrm{~b}$ & $20,69 \mathrm{ab}$ & $19,08 \mathrm{a}$ \\
$\mathrm{T} 4$ & $22,16 \mathrm{a}$ & $24,72 \mathrm{a}$ & $19,91 \mathrm{a}$ \\
\hline DMS & 4,62 & 5,38 & 5,66 \\
CV \% & 12,51 & 13,69 & 14,98 \\
\hline
\end{tabular}

Médias de tratamentos seguidas por letras distintas na coluna diferem pelo Teste tukey a $5 \%$ de significância

$\mathrm{Cv}=$ Coeficiente de variação; DMS= Diferença Mínima Significativa. 
A redução do tamanho dos poros é um dos indicativos da ocorrência do processo de compactação, haja vista que a macroporosidade se forma, sobretudo, a partir do estabelecimento de espaços entre as unidades estruturais do solo (MORAES et al., 2011).

Observou-se redução de macroporos do solo nos tratamentos $\mathrm{T} 3$ e $\mathrm{T} 2$, nas camadas $0-10 \mathrm{~cm}$ e $10-20 \mathrm{~cm}$, respectivamente. Beutler et al. (2006) observaram aumento na compactação em função do tráfego do trator sobre o solo, sendo a compactação verificada pelo aumento da resistência a penetração, da densidade e da microporosidade, reduzindo a quantidade de macroporos. Para os autores, macroporos apresentam-se menos resistentes e se deformam formando microporos que são mais resistentes suportando maiores pressões.

Moraes et al. (2011), avaliando a alteração da estrutura do solo gerada por níveis de compactação induzidos pelo tráfego de máquinas de um Latossolo Vermelho distroférrico manejado sob sistema plantio direto, verificaram que os valores de macroporosidade e microporosidade foram significativamente alterados pelos níveis de compactação induzidos pelo tráfego da colhedora.

$\mathrm{Na}$ tabela 3, verificam-se os resultados observados em relação à microporosidade do solo. Para as diferentes profundidades estudadas, não foi constatado diferenças estatísticas.

Tabela 3 - Microporos (\%) do solo em três profundidades referentes a testemunha e 3 níveis de compactação induzidas com trator.

\begin{tabular}{llll}
\hline Tratamentos & \multicolumn{3}{c}{ Profundidades } \\
\hline & $\mathbf{0 - 1 0} \mathbf{~ c m}$ & $\mathbf{1 0} \mathbf{- 2 0} \mathbf{~ c m}$ & $\mathbf{2 0} \mathbf{- 3 0} \mathbf{~ c m}$ \\
T1 & $34,31 \mathrm{a}$ & $31,52 \mathrm{a}$ & $36,11 \mathrm{a}$ \\
T2 & $35,39 \mathrm{a}$ & $38,13 \mathrm{a}$ & $36,23 \mathrm{a}$ \\
T3 & $40,92 \mathrm{a}$ & $32,82 \mathrm{a}$ & $39,20 \mathrm{a}$ \\
T4 & $35,04 \mathrm{a}$ & $34,59 \mathrm{a}$ & $36,92 \mathrm{a}$ \\
\hline DMS & 9,94 & 9,18 & 9,43 \\
CV $\%$ & 15,07 & 14,81 & 14,04 \\
\hline
\end{tabular}

Médias de tratamentos seguidas por letras distintas na coluna diferem pelo Teste tukey a $5 \%$ de significância

$\mathrm{Cv}=$ Coeficiente de variação; DMS= Diferença Mínima Significativa.

Streck et al. (2004) estudando o efeito de níveis de tráfego na alteração das propriedades físicas afetadas pela compactação no plantio direto, observaram que houve aumento na densidade e na resistência do solo à penetração, porém reduziu a porosidade total e a macroporosidade, e não comprometeu a microporosidade, o que também foi verificado nas análises de microporosidade do solo estudado.

Segundo Bergamin et al. (2010), estudando Latossolo Vermelho distroférrico, cultivado em sistema plantio direto, sob influência da compactação induzida pelo tráfego de trator agrícola, observaram que o tráfego de trator a partir de 2 passadas aumentam a compactação do solo, densidade do solo e reduzem a macroporosidade e porosidade total e quando o número de passadas for maior que 4, há aumento da resistência do solo à penetração de maneira efetiva em até 0,10 $m$ de profundidade.

A disponibilidade total de água do solo nas diferentes profundidades pode ser observada na tabela 4. 
Tabela 4 - Disponibilidade total de água do solo $(\mathrm{mm} / \mathrm{cm})$, em três profundidades referentes à testemunha e 3 níveis de compactação induzidas com trator.

\begin{tabular}{llll}
\hline Tratamentos & \multicolumn{3}{c}{ Profundidades } \\
\hline & $\mathbf{0} \mathbf{- 1 0} \mathbf{~ c m}$ & $\mathbf{1 0} \mathbf{- 2 0} \mathbf{~ c m}$ & $\mathbf{2 0} \mathbf{- 3 0} \mathbf{~ c m}$ \\
T1 & $1,30 \mathrm{a}$ & $1,31 \mathrm{ab}$ & $1,21 \mathrm{a}$ \\
$\mathrm{T} 2$ & $1,26 \mathrm{a}$ & $1,27 \mathrm{ab}$ & $1,21 \mathrm{a}$ \\
$\mathrm{T} 3$ & $1,22 \mathrm{a}$ & $1,35 \mathrm{a}$ & $1,21 \mathrm{a}$ \\
$\mathrm{T} 4$ & $1,25 \mathrm{a}$ & $1,18 \mathrm{~b}$ & $1,25 \mathrm{a}$ \\
\hline DMS & 0,21 & 0,15 & 0,15 \\
CV \% & 9,27 & 6,35 & 6,69 \\
\hline
\end{tabular}

Médias de tratamentos seguidas por letras distintas na coluna diferem pelo Teste tukey a $5 \%$ de significância

$\mathrm{Cv}=$ Coeficiente de variação; $\mathrm{DMS}=$ Diferença Mínima Significativa.

Verifica- se que para a primeira camada $(0-10 \mathrm{~cm})$ e para a última camada $(20-30 \mathrm{~cm})$ não houve diferença estatística entre os tratamentos. Porém, quando se analisou a camada de $10-20 \mathrm{~cm}$, pode-se verificar que $\mathrm{T} 4$ apresentou menor valor de disponibilidade total de água, sendo diferente do T3 e semelhante aos demais tratamentos, no entanto, T1, T2 e T3 foram considerados estatisticamente iguais.

A diminuição da disponibilidade de água no T4 na camada de $10-20 \mathrm{~cm}$ pode estar relacionada a compactação do solo, sendo comprometida pela alteração de sua estrutura, visto que o tratamento foi representado por 8 passadas com trator, maior intensidade de tráfego estudada. As alterações físicas, provocadas pela compactação, afetam o fluxo ou a concentração de água (STONE et al., 2006). Para Machado et al. (2005) no momento em que incide a pressão, o teor de água do solo, torna-se um fator determinante da intensidade de compactação.

A figura 1 apresenta a curva de retenção de água do solo, determinada pelos métodos da câmara de Richards e Psicrômetro WP4-T, ajustada para o modelo segundo Van Genuchen (1980).

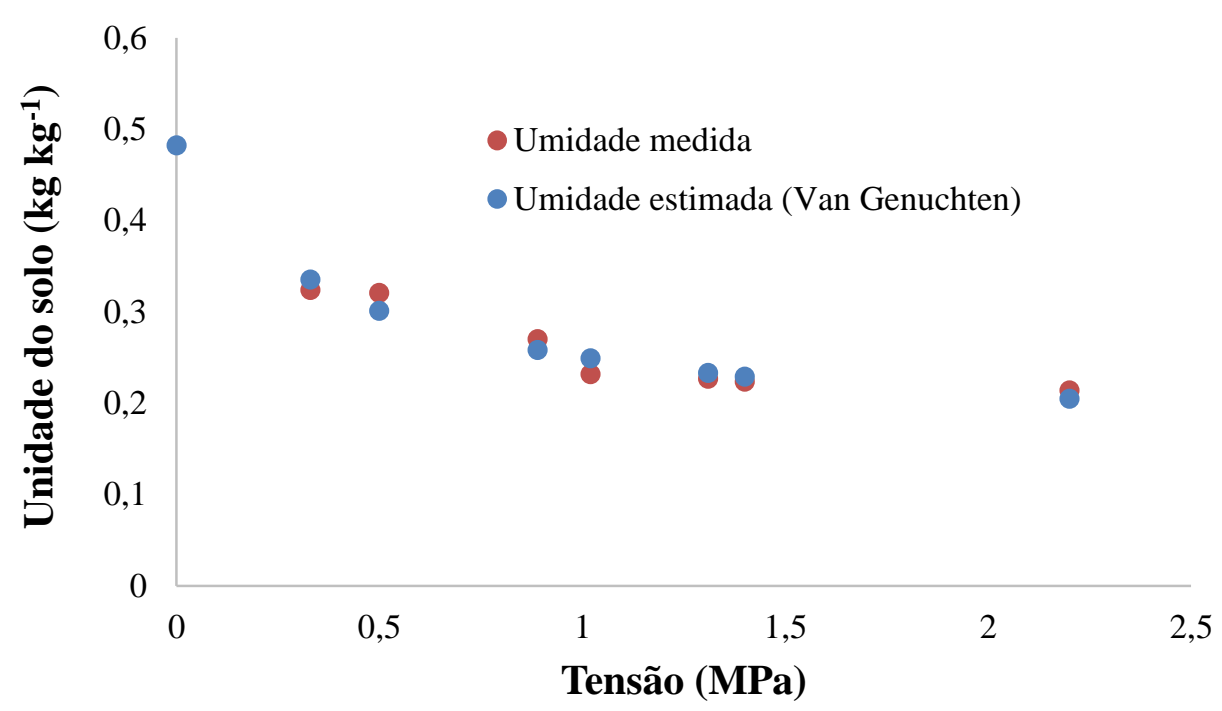

Figura 1 - Curva de retenção de água do solo ajustada pelo modelo Van Genutchen (1980), na camada $0-30 \mathrm{~cm}$. 
Pode-se observar na curva, que o maior valor de retenção de água foi observado próximo de 0,33 e o menor 0,21 . Observa-se também uma constância do teor de água em potenciais entre 1,50 a 2,25 Mpa.

A retenção de água no solo depende da granulometria e de algumas outras características do solo como estrutura e a fração mineralógica. As interações entre esses efeitos determinam a capacidade do solo reter e conduzir água. Segundo Libardi (2010), logo após a drenagem livre de um solo saturado no campo, as forças capilares são dominantes e à medida em que o solo seca, a adsorção adquire maior importância.

\section{CONCLUSÃO}

Conclui-se que a quantidade de passadas de um trator, em um Latossolo argiloso, alterou as características físicohídricas do solo, com exceção da microporosidade.

\section{REFERÊNCIAS}

BARRETO, H. B. F.; BATISTA, R. O.; FREIRE, F. G. C.; SANTOS, W. O.; COSTA, F. G. B. Análises de indicadores de retenção e armazenamento de água no solo do perímetro irrigado Gorutuba, em Janaúba-MG. Revista Verde, Mossoró, v.6, n.5, p.189-192, 2011.

BERGAMIN, A. C.; VITORINO, A. C. T.; FRANCHINI, J. C.; SOUZA, C. M. A. DE; SOUZA, R. R. DE. Compactação em um latossolo vermelho distroférrico e suas relações com o Crescimento radicular do milho. R. Bras. Ci. Solo, 34:681-691, 2010.

BEUTLER, A. N.; CENTURION, J. F.; CENTURION, M. A. P. C.; SILVA, A. P. DA. Efeito da compactação na produtividade de Cultivares de soja em latossolo vermelho. R. Bras. Ci. Solo, 30:787-794, 2006.
Nascimento (2009), afirma que, em solos arenosos os poros são maiores, em solos que restam pequenas quantidades de água em baixos potenciais matriciais são causados por rápido esvaziamento em elevados potenciais matriciais, o que explica a inclinação acentuada da CRA.

Cintra et al. (2004), também verificaram em um solo Argissolo Amarelo a queda da umidade do solo com aumento das sucções. Nas três camadas estudadas por ele, nas diferentes sucções aplicadas as CRA apresentaram formatos e comportamentos semelhante quanto à capacidade de retenção de água,

A camada de $10-20 \mathrm{~cm}$ foi a que apresentou maiores diferenças estatísticas, com alterações nos valores de $\mathrm{Pt}$, Macroporos e DTA.

BONINI, A. K.; SECCO, D.; SANTOS, R. F.; REINERT, D. J.; REICHERT, J. M. Atributos físico-hidricos e produtividade de trigo em um Latossolo sob estados de compactação. Ciência Rural, Santa Maria, v. 41, n. 9, p. 1543-1548, 2011.

CINTRA, F. L. D.; PORTELA, J. C.; NOGUEIRA, L. C. Caracterização física e hídrica em solos dos Tabuleiros Costeiros no distrito de irrigação Platô de Neópolis. Revista Brasileira de Engenharia Agrícola e Ambiental, 2004.

CRISPIM, F. A.; Compactação de Solos: Influencia de métodos e de parâmetros de compactação na estrutura dos solos. Viçosa - Minas Gerais - Brasil,2007.

DAVAlO, M. J. Curva de retenção de água no solo estimado pelo método da câmara de Richards e Psicrômetro. 2013. Dissertação (Mestrado) Universidade Estadual Paulista, Faculdade 
de Ciências Agrárias e Veterinárias de Jaboticabal, 2013.

EMBRAPA - Empresa Brasileira de Pesquisa Agropecuária. Serviço Nacional de Levantamento e Conservação de Solos. Manual de métodos de análise do solo. Rio de Janeiro: EMBRAPA. 1997, 212 p.

EMBRAPA. Manual de métodos de análise de solos. Rio de Janeiro: Embrapa solos, 2011. 230 p. (Documentos 132). Disponível em: <http://www.cnps.embrapa.br/publicacoes >. Acesso em 01 de maio de 2016.

EMBRAPA. Sistema brasileiro de classificação de solos. 3. ed. Rio de Janeiro, Centro Nacional de Pesquisa de Solos, 2013.

GRIGOLON, G. B Curva de retenção de água no solo determina a partir de um número de mínimo de pares de umidade e tensão na câmara de Richards. Dissertação (Mestrado) - Universidade de São Paulo. Escola Superior de Agricultura "'Luiz de Queiroz". Piracicaba, 2013.

GUBIANI, P. I. Regularidade de resposta da Cultura do milho à compactação do solo. Dissertação (Doutorado). Universidade Federal de Santa Maria (UFSM, RS). Programa de Pós-Graduação em Ciência do Solo, Área de Concentração em Processos Físicos e Morfogenéticos do Solo. Santa Maria, RS, 2012.

KLEIN, V. A.; BASEGGIO, M.; MADALOSSO, T.; MARCOLINI, C. D. Textura do solo e a estimativa do teor de água no ponto de murcha permanente com psicrômetro. Ciência Rural, Santa Maria, v.40, n.7, p.1550-1556, 2010.

KÖPPEN, W. 1936. Das geographics System der Klimate.Gebr, Borntraeger, $1-44$.

LIBARDI, P. L. Água no solo. In: VAN LIER, Q. de J. Física do solo. Viçosa:
Sociedade Brasileira de Ciência do solo, 2010.

MACHADO, A. L. T.; REIS, A. V. dos; FERREIRA, M. F. P.; MACHADO, R. L. T.; MACHADO, A. L. C.; BAUER, G. B. Influência da pressão de inflação do pneu do trator na resistência do solo à penetração. R. bras. Agrociência, Pelotas, v. 11, n. 4, p. 481-486, out-dez, 2005.

MORAES, $\quad$ M. T.; DEBIASI, H.; FRANCHINI, J. C.; SILVA, V. R. Propriedades físicas do solo sob diferentes níveis de compactação em um latossolo vermelho distroférrico. Embrapa Soja. Documentos, 2011. (EMBRAPA Soja. Documento 328).

NASCIMENTO, P. dos S. Análise do uso da curva de retenção de água no solo determinada por diferentes métodos e planilha para manejo da irrigação. 2009. 97 f. Dissertação (Mestrado em Ciências Agrárias) - Centro de Ciências Agrárias, Ambientais e Biológicas, Universidade Federal do Recôncavo da Bahia, Cruz das Almas, 2009.

PASSOS, J. F. M. Atributos do solo e produtividade da soja em um Latossolo Bruno afetados por sistemas de manejo e calagem. 2004. 79 f. Dissertação (Mestrado em Ciência do Solo) - Centro de Ciências Agroveterinárias, Universidade do Estado de Santa Catarina, Santa Catarina, 2004.

REINERT, D. J.; REICHERT, J. M. Propriedades físicas do solo. Santa Maria, 2006.

SILVA, F. de A. S.; AZEVEDO, C. A. V. de. The Assistat Software Version 7.7 and its use in the analysis of experimental data. Afr. J. Agric. Res, v.11, n.39, p.37333740, 2016.

STONE, L. F.; SILVEIRA, P. M.; MOREIRA, J. A. A. Atributos físicohídricos do solo sob plantio direto. Santo Antônio de Goiás: Embrapa Arroz e 
Feijão, 2006. 39p. (Embrapa Arroz e Feijão. Documentos, 191).

STRECK, C. A.; REINERT, D. J.; REICHERT, J. M.; KAISER, D. R. Modificações em propriedades físicas com a compactação do solo causada pelo tráfego induzido de um trator em plantio direto. Cienc. Rural, Santa Maria, v. 34, n. 3, p. 755-760, Jun., 2004.

VAN GENUCHTEN, M. T. A closed-form equation for predicting the hydraulic conductivity of unsaturated soils. Soil Science Society of American Journal, Madison, WI, v. 44, p. 892- 898, 1980. 